\title{
LIQUID PUMPING AND MIXING BY PZT SYNTHETIC JET
}

\author{
Luan Le Van ${ }^{1}$, Cuong Nguyen Nhu ${ }^{1}$, Tuan Nguyen Tai ${ }^{2}$, Thien Xuan Dinh ${ }^{3}$, Canh-Dung Tran ${ }^{4}$, \\ Lam Dang Bao ${ }^{2}$, Tung Thanh Buil, Van Thanh Dau ${ }^{5}$, Trinh Chu Duc ${ }^{1 *}$ \\ ${ }^{1}$ VNU University of Engineering and Technology, Hanoi, VIETNAM \\ ${ }^{2}$ School of Mechanical Engineering, Hanoi University of Science and Technology, Hanoi, VIETNAM \\ ${ }^{3}$ Graduate School of Science and Engineering, Ritsumeikan University, Shiga, JAPAN \\ ${ }^{4}$ School of Mechanical and Electrical Engineering, University of Southern Queensland, AUSTRALIA \\ ${ }^{5}$ School of Engineering and Built Environment, Griffith University, Queensland, AUSTRALIA
}

\begin{abstract}
In this paper, a PZT synthetic jet that can function as both an efficient pumping and mixing device is developed. Compare with the conventional design where the practice of controlling the internal flow is undertaken by microvalves structure, this approach promotes the durability and allows the device to work with different liquids at high Reynold number without losing of backflow from the diffuser, therefore provides efficient mixing. The pumping performance is applicable for commercialized counterparts while the homogeneous medium was obtained at downstream in the experiments, which was further confirmed by simulation. Notably, the chaotic mixing feature of the device is also applicable for immiscible liquids with the micro-droplet formation result at the outlet.
\end{abstract}

\section{KEYWORDS}

Liquid pumping, liquid mixing, PZT synthetic jet.

\section{INTRODUCTION}

Various application involving microfluidic devices for pumping, mixing and sensing has played significant role in research and development nowadays [1]-[6]. Figure 1 illustrates the involved steps in a cell counting systems for microfluidic biosensing. Not only do cells need to be successively conveyed through different part of the systems, it also needs to be mixed with functional solution or particles for counting and manipulating purpose. For example, the blood sample is required to be mixed with lysis solution in order to remove undesired red blood cells. Therefore, recent researches have attempted to develop a multifunctional device which are capable of both pumping and mixing in order not to use several or bulky parts in these systems. The practice of controlling the internal flow in the pumping and mixing device usually involves the use of the microvalve structure. Pumping device with this flow controlling mechanism, however, suffers from wear and fatigue, high-pressure drop across the valve. To address this problem, valveless pump [7]-[12] was proposed. These types of pump do not use the moving part as a flow regulating mechanism but use the fixed-geometry structure instead. Thanks to this flow regulating mechanism, micropump-micromixer can avoid those issues that are usually encountered in the device with moving-parts while enjoying a simple design.

Based on our previous development of a valveless micropump [13]-[18] we here report a valveless synthetic jet. Unlike the nozzle/diffuser structure where they connect to a pump chamber at two separated positions, the pump chamber sealed at one end is linked to an emitting nozzle at another end without any used diffuser in our work. Therefore, our device allows pumping different liquids at high Reynold number without losing of backflow from the diffuser, therefore providing efficient mixing.

\section{DESIGN}

The mechanism of the proposed liquid pumping and mixing approach is illustrated in Figure 2(a). The pump chamber as the driving force source is sealed with PZT diaphragm and is only linked to an emitting nozzle at one end. The pumping/mixing nozzle connects the pump chamber and the outlet. The voltage placed on the PZT diaphragm produces strong vibrations on the diaphragm. Such vibrations create a driving force on the fluid due to pressure changes in the chamber. This allows the chamber

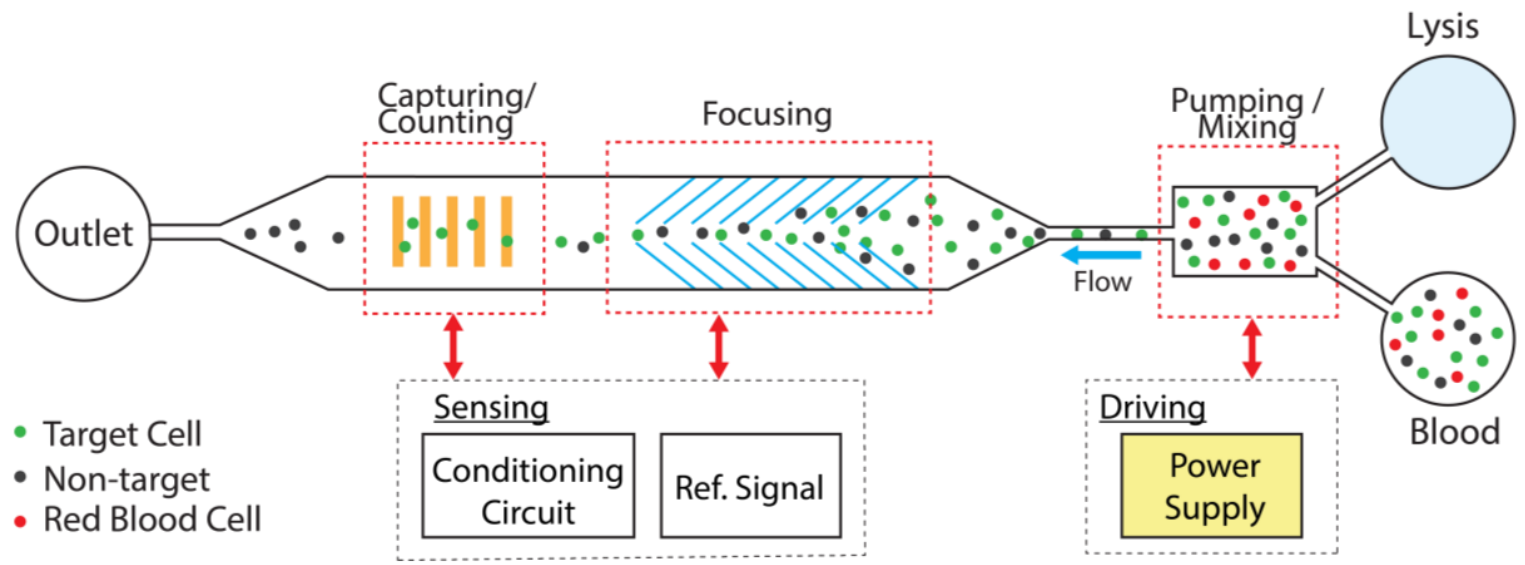

Figure 1: Microfluidic bio sensing system. Liquid pumping and mixing are required for enabling the operation of the system. 


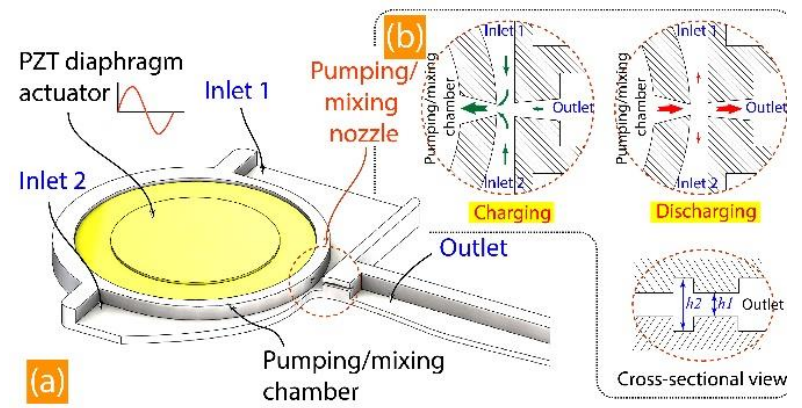

Figure 5: Proposed device. (a) Schematic view of device, (b) pumping mechanism at nozzle.

to repeatedly charge the liquid from both inlets and discharge them through the junction as in Figure 2(b). The nozzle also lies at the region where two inlet channels intersect. As being drawn to the pump chamber, the inflow currents meet and collide at the pumping/mixing nozzle. Afterward, both flows are fed into the pump chamber, where the circular shape conveniently helps generate vortexes to mix flows. This allows the device to act as both a liquid pumping and mixing device. The successive charge/discharges phase periodically draws and push mixed liquid through the outlet nozzle.

The proposed device can be coupled with the capacitive sensor structure. The electrodes are placed along the outlet of the micropump. These electrodes form a capacitor with the pumping liquid as the dielectric medium. The capacitance measured from the electrodes depends on the permittivity of the medium. Since liquids have different dielectric permittivity values, that value of the mixture also varies depending on the mixing ratio. The capacitance of the sensor, thus, can serve as an identification for the fluid and the current mixing ratio.

\section{SIMULATION SETUP}

A numerical modelling is conducted using COMSOL Multiphysics to simulate the flow of the liquid. A 3-D model as well as its meshing result is shown Figure 3(a). The PZT diaphragm is actuated by a time-dependent sinusoidal voltage $V_{a p p}=V_{o} \sin (2 \pi f \times t)$, with $\boldsymbol{V}_{\mathbf{0}}$ the amplitude of the applied voltage and $\boldsymbol{f}$ the driving frequency. The flow with the charge and discharge phases

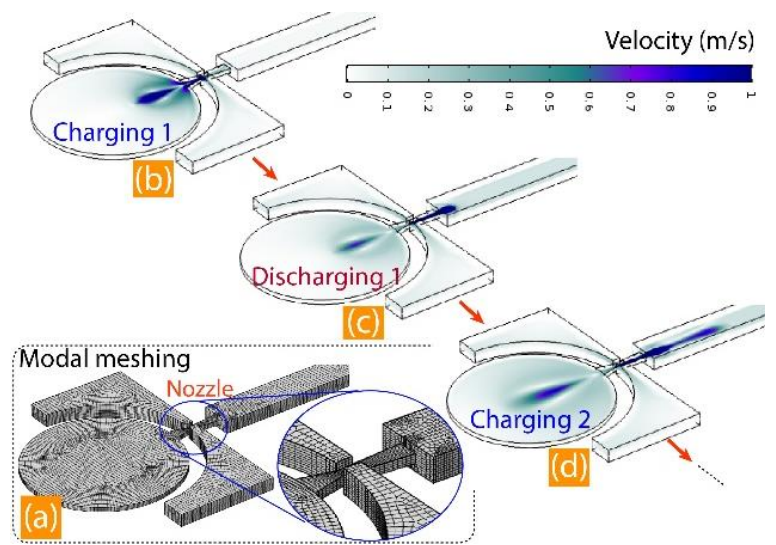

Figure 4: Simulated flow presented high velocity appearing inside the pump chamber and outlet channel. are presented in Figure 3(b), (c) and (d). It can be clearly seen from the velocity profile that a flow at high velocity appears inside the pump chamber and outlet channel, indicating the liquid's being drawn and discharged at the corresponding phase of the pumping/mixing phase. Each charge/discharge circle generates a net flow into the outlet channel.

\section{EXPERIMENT}

Figure 4 experimentally depicts the pumping effect of a prototype, made by addictive manufacturing with printing step of 16 microns. The rising of the liquid level inside the pump indicates a net driving force created by the pump chamber together with the PZT diaphragm,

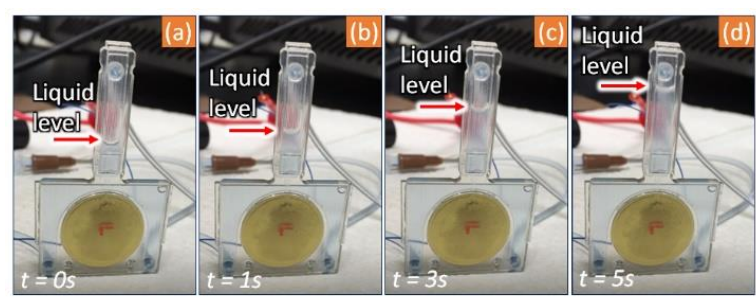

Figure 2: Experiment confirm the pumping performance. liquid level demonstrates a net driving force created by the pumping chamber.
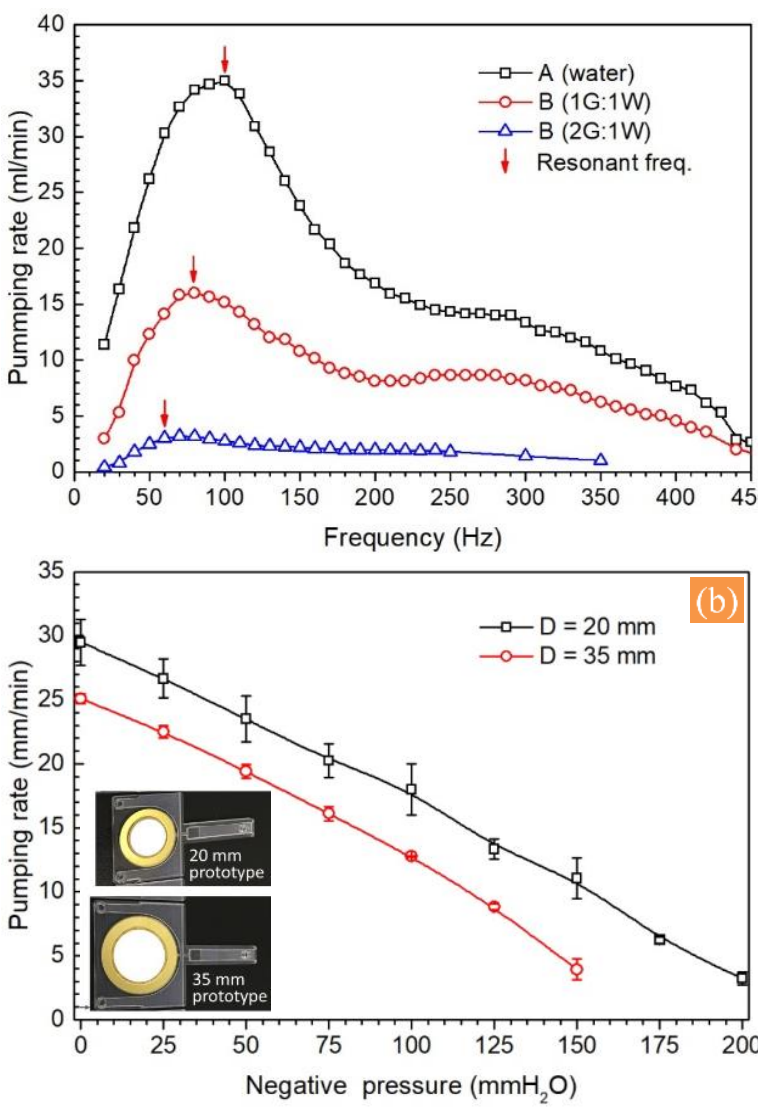

Figure 3: Pumping performance. (a) Pumping rate of different liquids with driving voltage VPZT $=220 \mathrm{~V}$. The glycerin-water mixture B (glyceryl:water $1: 1 \mathrm{v} / \mathrm{v}$ ) and (glyceryl : water 1:1 v/v) (b) Effect of the PZT diaphragm diameter on the pump's water $Q-P$ character. 
confirming the pumping performance of the device. Pumping-rate versus driving frequency is presented in Figure 5(a). For the lower part of the frequency range, there is a linear dependence between the obtained flow rate and the frequency. As the driving frequency is high, the flow rate decreases and gradually becomes trivial. A flow rate of $35 \mathrm{ml} / \mathrm{min}$ was achieved at a resonance frequency $100 \mathrm{~Hz}$ for water. This pumping performance is applicable for commercialized counterparts [19], [20].

The ability of pumping liquids of various viscosities is also described in Figure 5(a) where the dependence of resonant frequency and pump flowrate with solution parameters is observed. It can be observed that the resonant frequency and flowrate of the pump decrease with the increase of viscosity and the density. A shift of resonant frequency corresponds to the rise of the liquid mass inertia which makes the liquid less responsive to the movement from the PZT membrane. The higher the viscosity is, the lower is the frequency required to synchronize with the membrane motion. The effect of PZT membrane to pumping performance in Figure 5(b) shows a backpressure-flowrate more than $2 \mathrm{kPa}$ achieved by our device in spite of its valveless structure.

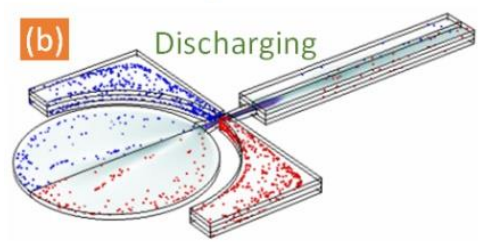

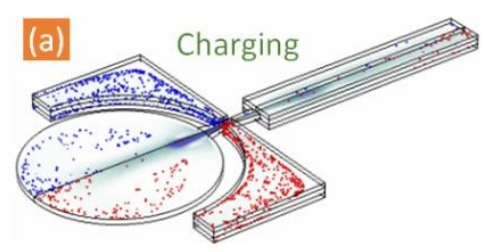

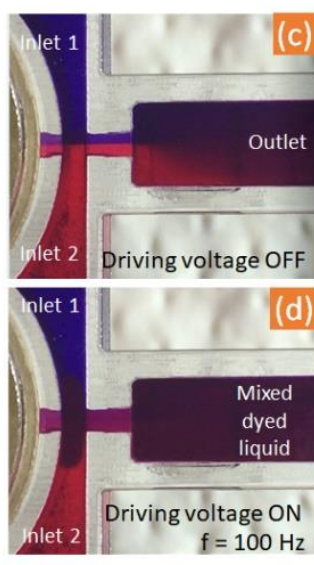

Figure 6: Mixing predicted by simulation. (a), (b): Simulation - mixing at 0.2ms and 0.5ms. (c), (d): Experiment with miscible colored liquids, the liquid was homogenously mixed.

Mixing performance of the device is transiently simulated as shown in Figure 6(a, b) by particle tracing simulation. Virtual particles of different colors are injected to both inlets. Particles from two inlets are transported toward the intersection, where they collide and started to be mixed. The circular patterns of the traced particles inside the chamber also indicate the formation of vortices. Therefore, the fluids are mixed at both the nozzle intersection and the pumping/mixing chamber. The mixing effects can be clearly seen at the outlet. The simulation is further confirmed by experiments using differently dyed liquid as shown in Figure 6(c), (d). The shade of the fluid indicates the mixing efficacy. It can be clearly seen that without the driving voltage, the flows the two dyed liquids do not show any mixing effect at the upstream of the outlet or the region near the emitting nozzle as in Figure 6(c). The mixing effect is only experienced at the lower end of the outlet, which is attributed to the diffusion along the contact surface of the two flows. However, strong collisions of flows included by the PZT diaphragm movement create chaotic mixing at the nozzle and the liquids were instantly mixed into the homogeneous medium at the outlet as in Figure 6(d).

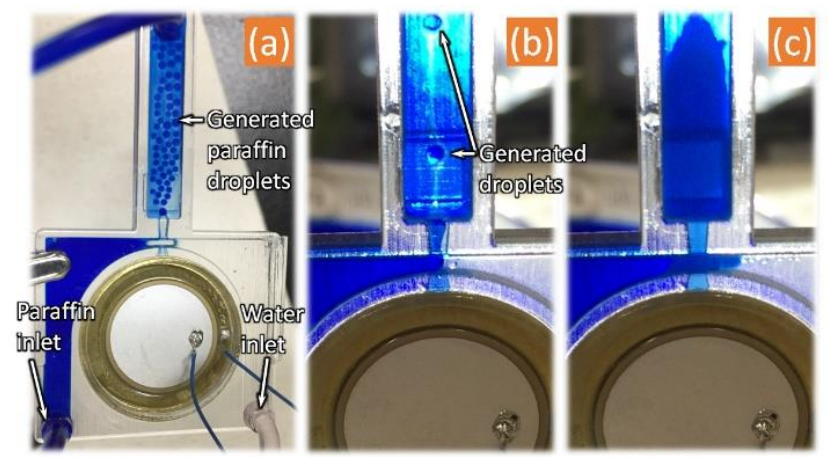

Figure 7: Experiment with immiscible (water - dyed paraffin), the immiscible liquids are mixed into micro droplet.

Notably, chaotic mixing is applicable for even immiscible liquids. One inlet of the device was fed with dyed paraffin while the other is connect to water source as shown in Figure 7. They are pumped, mixed and the paraffin current are broken into micro-droplets of different controlled sizes after exiting the emitting nozzle. Thus, the proposed device also has the potential to act as a microdroplet generator device.

We further monitor the pumping - mixing by the capacitance sensors embedded downstream the mixing zone, along the outlet channel. This sensor is in charge of measuring the capacitance along the outlet channel for different fluid materials and mixing ratio. Figure 8 shows the output of the sensor using $\mathrm{NaCl}$ solution and deionized water as test liquids introduced to the device at each inlet. The capacitive sensors was tested with the solution of ratio ranging from $0.5 \%$ to $4.5 \%$ (wt:wt $\%$ ). A linear dependence was obtained with the highest capacitance change of $1.6 \mathrm{pF}$ for $3.8 \%$ sodium chloride concentration. The results confirm the ability to develop a fully automated pumping sensing mixer for bio medical applications.

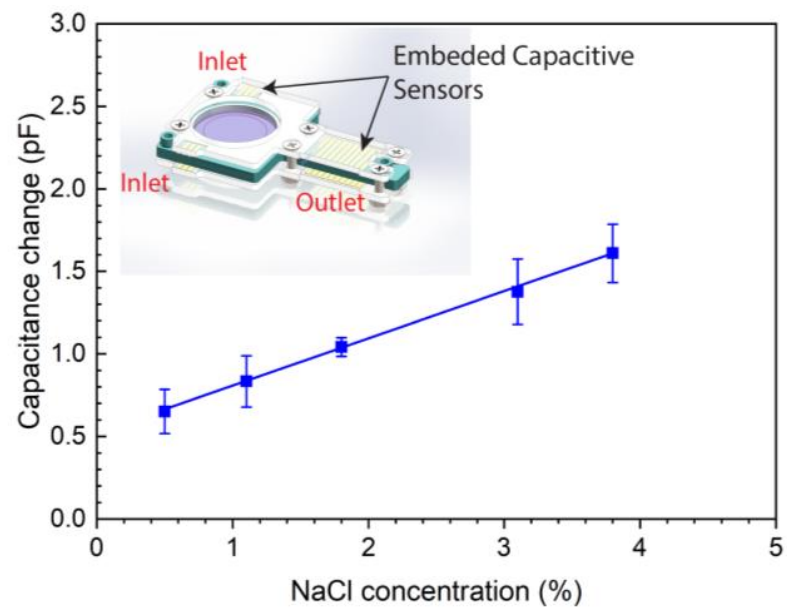

Figure 8: Mixing performance evaluation with embedded capacitive sensor at the output. 


\section{CONCLUSION}

A synthetic jet device has been developed based on the combination of valveless structure and PZT diaphragm for the liquid pumping and mixing application. PZT diaphragm through its deformation creates a driving force on the fluid due to pressure changes in the chamber, allowing the chamber to charge/discharge fluid through the emitting nozzle. Since the device do not use the valve as the flow controlling mechanism, it is less likely to be suffered from wear and fatigue while enjoying the simple structure. The experiment and further simulation results has confirmed the performed of the devices and its properties. The one-gate pump chamber and the opposite inlet channels provide the proposed device with the ability to pump and mix the fluid simultaneously. It is noteworthy that the chaotic mixing effect is also applicable for immiscible liquid inputs with micro-droplets formation results. The results acquired from the capacitive sensor embedded along the outlet channel demonstrate the potential for a fully automated pumping - sensing mixer.

\section{ACKNOWLEDGEMENTS}

We would like to acknowledge the Vietnamese Ministry of Science and Technology (MOST) for financial support through a project entitled "Development on the aptamer-based and electrical impedance sensing microfluidic system for circulating tumor cells detection", project code NDT.15.TW/16.

\section{REFERENCE}

[1] F. Abhari, H. Jaafar, and N. A. Md Yunus, A comprehensive study of micropumps technologies, vol. 7, no. 10. 2012.

[2] P. T. Hoa, T. X. Dinh, and V. T. Dau, "Design Study of Multidirectional Jet Flow for a Triple-Axis Fluidic Gyroscope," IEEE Sens. J., vol. 15, no. 7, pp. 41034113, Jul. 2015.

[3] L. Q. Do et al., "Dielectrophoresis Microfluidic Enrichment Platform with Built-In Capacitive Sensor for Rare Tumor Cell Detection," BioChip J., Apr. 2018.

[4] V. T. Dau, T. X. Dinh, T. T. Bui, and T. Terebessy, "Bipolar corona assisted jet flow for fluidic application," Flow Meas. Instrum., vol. 50, pp. 252 260, 2016.

[5] T. X. Dinh, D. B. Lam, C. D. Tran, T. T. Bui, P. H. Pham, and V. T. Dau, "Jet flow in a circulatory miniaturized system using ion wind," Mechatronics, vol. 47, no. September, pp. 126-133, Nov. 2017.

[6] L. Q. Do, T. T. Bui, H. T. T. Tran, K. Kikuchi, M. Aoyagi, and T. C. Duc, "Fluidic platform with embedded differential capacitively coupled contactless conductivity detector for micro-object sensing," Int. J. Nanotechnol., vol. 15 , no. 1/2/3, p. 24, 2018.

[7] S. Yang, X. He, S. Yuan, J. Zhu, and Z. Deng, “A valveless piezoelectric micropump with a Coanda jet element," Sensors Actuators, A Phys., vol. 230, pp. 74-82, 2015.

[8] C. Yamahata, C. Lotto, E. Al-Assaf, and M. a M. Gijs,
"A PMMA valveless micropump using electromagnetic actuation," Microfluid. Nanofluidics, vol. 1, no. 3, pp. 197-207, 2005.

[9] K. Tanaka, V. T. Dau, R. Sakamoto, T. X. Dinh, D. V. Dao, and S. Sugiyama, "Fabrication and Basic Characterization of a Piezoelectric Valveless Micro Jet Pump," Jpn. J. Appl. Phys., vol. 47, no. 11, pp. 86158618, Nov. 2008.

[10] V. T. Dau, T. X. Dinh, T. Katsuhiko, and S. Susumu, "A cross-junction channel valveless-micropump with PZT actuation," Microsyst. Technol., vol. 15, no. 7, pp. 1039-1044, May 2009.

[11] V. T. Dau, T. X. Dinh, C. D. Tran, P. N. Bui, D. D. Vien, and H. T. Phan, "Fluidic mechanism for dualaxis gyroscope," Mech. Syst. Signal Process., vol. 108, pp. 73-87, 2018.

[12] T. X. Dinh, V. T. Dau, S. Sugiyama, and P. H. Pham, "Fluidic device with pumping and sensing functions for precise flow control," Sensors Actuators, B Chem., vol. 150 , no. 2, pp. 819-824, Oct. 2010.

[13] N. Q. Dich, T. X. Dinh, P. H. Pham, and V. T. Dau, "Study of valveless electromagnetic micropump by volume-of-fluid and OpenFOAM," Jpn. J. Appl. Phys., vol. 057201, no. 5, p. 057201, May 2015.

[14] V. T. Dau, T. X. Dinh, Q. D. Nguyen, R. Amarasinghe, K. Tanaka, and S. Sugiyama, "Microfluidic valveless pump actuated by electromagnetic force," Proc. IEEE Sensors, no. Type II, pp. 679-682, 2009.

[15] T. X. Dinh, N. T. M. Le, V. T. Dau, and Y. Ogami, “A dynamic model for studying valveless electromagnetic micropumps," J. Micromechanics Microengineering, vol. 21, no. 2, p. 025015, Feb. 2011.

[16] V. T. Dau, T. X. Dinh, and T. T. Bui, "Jet flow generation in a circulatory miniaturized system," Sensors Actuators B Chem., vol. 223, pp. 820-826, 2015.

[17] V. T. Dau and T. X. Dinh, "Numerical study and experimental validation of a valveless piezoelectric air blower for fluidic applications," Sensors Actuators B Chem., vol. 221, pp. 1077-1083, Jul. 2015.

[18] Tung Thanh Bui, Thien Xuan Dinh, Phan Thanh Hoa, and Van Thanh Dau, "Study on the PZT diaphragm actuated multiple jet flow in a circulatory miniaturized system," in 2015 IEEE SENSORS, 2015, pp. 1-4.

[19] Takasago fluidics micropump, "Piezoelectric Micro Pump - Takasago Fluidic Systems." [Online]. Available: http://www.takasagofluidics.com/products/products_pump/transfer/. [Accessed: 09-Sep-2018].

[20] Curie Jet Micropump, “CurieJet ${ }^{\circledR}$ Liquid/Gas Micro Pump :: Micro Fluidics Pump, Micropump.” [Online]. Available: http://www.curiejet.com/en/products/list.php?pin=09 9861a6e97183485912f85df4e401fd\&type=s. [Accessed: 09-Sep-2018].

\section{CONTACT}

*Trinh Chu Duc, tel: $\quad+84-936686156$ trinhcd@vnu.edu.vn. 